Maltepe Journal of Mathematics

ISSN:2667-7660, URL:HTTP://DERGIPARK.ORG.TR/TR/PUB/MJM

Volume III Issue 2 (2021), PAges 74-90. Doi:HTtPs://DOI.ORG/10.47087/MJM.926078

\title{
SOME CHARACTERIZATIONS ON GEODESIC, ASYMPTOTIC AND SLANT HELICAL TRAJECTORIES ACCORDING TO PAFORS
}

\author{
KAHRAMAN ESEN ÖZEN* AND MURAT TOSUN** \\ *SAKARYA, TURKEY, ORCID ID: 0000-0002-3299-6709 \\ **DEPARTMENT OF MATHEMATICS, FACULTY OF SCIENCE AND ARTS, SAKARYA \\ UNIVERSITY, SAKARYA, TURKEY, ORCID ID: 0000-0002-4888-1412
}

\begin{abstract}
In this paper, we study the geodesic, asymptotic, and slant helical trajectories according to PAFORS in three-dimensional Euclidean space and give some characterizations on them. Also, we explain how we determine the helix axis for slant helical trajectories (according to PAFORS). Moreover, we develop a method that enables us to find the slant helical trajectory (if exists) lying on a given implicit surface which accepts a given fixed unit direction as an axis and a given angle as the constant angle. This method also gives information when the desired slant helical trajectory does not exist. The results obtained here involve some differential and partial differential equations or they are based on these equations. The aforementioned results are new contributions to the field and they may be useful in some specific applications of particle kinematics and differential geometry.
\end{abstract}

\section{Introduction and Preliminaries}

Despite its long history, the theory of surfaces is still an issue of interest in 3dimensional Euclidean space. Darboux frame, which is constructed on a surface, is an important part of this theory. It exists at all the points of a curve on a regular surface [1]. The success of developing this frame belongs to French mathematician J. G. Darboux. From the discovery of this frame until now, many researchers have presented lots of interesting studies on the theory of surfaces by using this frame. Some of these studies can be found in $[277$. One of the newest of them is the study 8 presented by Özen and Tosun. They introduced PAFORS (positional adapted frame on the regular surface) for the trajectories with non-vanishing angular momentum in this study.

Let the Euclidean 3 -space $E^{3}$ be taken into account with the standard scalar product $\langle\mathbf{N}, \mathbf{P}\rangle=n_{1} p_{1}+n_{2} p_{2}+n_{3} p_{3}$ where $\mathbf{N}=\left(n_{1}, n_{2}, n_{3}\right), \mathbf{P}=\left(p_{1}, p_{2}, p_{3}\right)$ are any vectors in $E^{3}$. The norm of $\mathbf{N}$ is given as $\|\mathbf{N}\|=\sqrt{\langle\mathbf{N}, \mathbf{N}\rangle}$. If a differentiable

2020 Mathematics Subject Classification. 70B05; 53A05.

Key words and phrases. kinematics of a particle; regular surfaces; slant helix; differential equations; initial value problem.

(C)2019 Maltepe Journal of Mathematics.

Submitted on April 22 th. 2021, Published on October 30 th. 2021.

Communicated by Fuat USTA. 
curve $\alpha=\alpha(s): I \subset \mathbb{R} \rightarrow E^{3}$ satisfies $\left\|\frac{d \alpha}{d s}\right\|=1$ for all $s \in I$, it is called a unit speed curve. In that case, $s$ is said to be arc-length parameter of $\alpha$. A differentiable curve is called a regular curve if its derivative does not equal zero along the curve. An arbitrary regular curve can be reparameterized by the arc-length of itself 9 . Throughout the paper, the differentiation with respect to the arc-length parameter $s$ will be shown with a dash.

Assume that a point particle moves along the trajectory $\alpha: I \subset R \rightarrow M \subset E^{3}$ which is a unit speed curve and lies on a regular surface $M$. In this case, there exists Darboux frame denoted by $\{\mathbf{T}, \mathbf{Y}, \mathbf{U}\}$ along the trajectory $\alpha=\alpha(s)$. Here, $\mathbf{T}$ is the unit tangent vector of $\alpha, \mathbf{U}$ is the unit normal vector of $M$ restricted to $\alpha$ and $\mathbf{Y}$ is the unit vector given by $\mathbf{Y}=\mathbf{U} \times \mathbf{T}$. The derivative formulas of Darboux frame are given by:

$$
\left(\begin{array}{c}
\mathbf{T}^{\prime} \\
\mathbf{Y}^{\prime} \\
\mathbf{U}^{\prime}
\end{array}\right)=\left(\begin{array}{ccc}
0 & k_{g} & k_{n} \\
-k_{g} & 0 & \tau_{g} \\
-k_{n} & -\tau_{g} & 0
\end{array}\right)\left(\begin{array}{c}
\mathbf{T} \\
\mathbf{Y} \\
\mathbf{U}
\end{array}\right)
$$

The functions $\tau_{g}, k_{g}$ and $k_{n}$ are called geodesic torsion, geodesic curvature and normal curvature of the curve $\alpha$, respectively 1,2$]$. The following relationships based on these functions are well known [1]:

(1) $\alpha=\alpha(s)$ is an asymptotic curve if and only if $k_{n}=0$,

(2) $\alpha=\alpha(s)$ is a geodesic curve if and only if $k_{g}=0$.

Another thing that can be of importance is the angular momentum vector of the aforementioned particle about the origin. This vector has an important place in particle kinematics. It is calculated as the vector product of the position vector and linear momentum vector of the particle. It always lies on the instantaneous plane $S p\{\mathbf{Y}(s), \mathbf{U}(s)\}$. Let us assume that this vector never equals zero during the motion of the aforementioned particle. This assumption ensures that the functions $\langle\alpha(s), \mathbf{Y}(s)\rangle$ and $\langle\alpha(s), \mathbf{U}(s)\rangle$ are not zero at the same time along the trajectory $\alpha=\alpha(s)$. That is, we can say that the tangent line of $\alpha=\alpha(s)$ at any point does not pass through the origin. In this case, there exists PAFORS denoted by $\{\mathbf{T}(s), \mathbf{G}(s), \mathbf{H}(s)\}$ along the trajectory $\alpha=\alpha(s)$. As mentioned earlier, this frame has been recently introduced in the study 8. PAFORS contains a lot of information about the position vector of the moving particle. Also, it enables us to study together the kinematics of the moving particle on surface, the differential geometry of the trajectory and the differential geometry of the surface. So, it is expected that PAFORS will enable more convenient observation environment for the researchers studying on modern robotics in the future. In the system $\{\mathbf{T}(s), \mathbf{G}(s), \mathbf{H}(s)\}, \mathbf{T}(s)$ is the unit tangent vector of the trajectory and it is the common base vector of this frame with the Darboux frame. Consider the vector whose starting point is the foot of the perpendicular (from $O$ to instantaneous plane $S p\{\mathbf{T}(s), \mathbf{U}(s)\}$ ) and the endpoint is the foot of the perpendicular (from $O$ to instantaneous plane $S p\{\mathbf{T}(s), \mathbf{Y}(s)\})$. The equivalent of this vector at the point $\alpha(s)$ determines the third base vector $\mathbf{H}(s)$ of PAFORS. Therefore, $\mathbf{H}(s)$ is calculated as follows (see 8 for more details):

$$
\mathbf{H}(s)=\frac{\langle-\alpha(s), \mathbf{Y}(s)\rangle}{\sqrt{\langle\alpha(s), \mathbf{Y}(s)\rangle^{2}+\langle\alpha(s), \mathbf{U}(s)\rangle^{2}}} \mathbf{Y}(s)+\frac{\langle\alpha(s), \mathbf{U}(s)\rangle}{\sqrt{\langle\alpha(s), \mathbf{Y}(s)\rangle^{2}+\langle\alpha(s), \mathbf{U}(s)\rangle^{2}}} \mathbf{U}(s) .
$$


On the other hand, the second base vector $\mathbf{G}(s)$ is obtained by vector product $\mathbf{H}(s) \wedge \mathbf{T}(s)$ as in the following:

$$
\mathbf{G}(s)=\frac{\langle\alpha(s), \mathbf{U}(s)\rangle}{\sqrt{\langle\alpha(s), \mathbf{Y}(s)\rangle^{2}+\langle\alpha(s), \mathbf{U}(s)\rangle^{2}}} \mathbf{Y}(s)+\frac{\langle\alpha(s), \mathbf{Y}(s)\rangle}{\sqrt{\langle\alpha(s), \mathbf{Y}(s)\rangle^{2}+\langle\alpha(s), \mathbf{U}(s)\rangle^{2}}} \mathbf{U}(s) .
$$

Since tangent vector $\mathbf{T}(s)$ is mutual in both PAFORS and Darboux frame, the vectors $\mathbf{Y}(s), \mathbf{U}(s), \mathbf{G}(s)$ and $\mathbf{H}(s)$ lie on the same plane. Thus, there is a relation between PAFORS and Darboux frame as follows:

$$
\left(\begin{array}{l}
\mathbf{T}(s) \\
\mathbf{G}(s) \\
\mathbf{H}(s)
\end{array}\right)=\left(\begin{array}{ccc}
1 & 0 & 0 \\
0 & \cos \Omega(s) & -\sin \Omega(s) \\
0 & \sin \Omega(s) & \cos \Omega(s)
\end{array}\right)\left(\begin{array}{c}
\mathbf{T}(s) \\
\mathbf{Y}(s) \\
\mathbf{U}(s)
\end{array}\right)
$$

where $\Omega(s)$ is the angle between the vectors $\mathbf{U}(s)$ and $\mathbf{H}(s)$ (or likewise $\mathbf{Y}(s)$ and $\mathbf{G}(s)$ ) which is positively oriented from $\mathbf{U}(s)$ to $\mathbf{H}(s)$ (or likewise from $\mathbf{Y}(s)$ to $\mathbf{G}(s)$ )(see Figure 1). Additionally, the derivative formulas of PAFORS are given by

$$
\left(\begin{array}{l}
\mathbf{T}^{\prime}(s) \\
\mathbf{G}^{\prime}(s) \\
\mathbf{H}^{\prime}(s)
\end{array}\right)=\left(\begin{array}{ccc}
0 & k_{1}(s) & k_{2}(s) \\
-k_{1}(s) & 0 & k_{3}(s) \\
-k_{2}(s) & -k_{3}(s) & 0
\end{array}\right)\left(\begin{array}{c}
\mathbf{T}(s) \\
\mathbf{G}(s) \\
\mathbf{H}(s)
\end{array}\right)
$$

where

$$
\begin{aligned}
& k_{1}(s)=k_{g}(s) \cos \Omega(s)-k_{n}(s) \sin \Omega(s) \\
& k_{2}(s)=k_{g}(s) \sin \Omega(s)+k_{n}(s) \cos \Omega(s) \\
& k_{3}(s)=\tau_{g}(s)-\Omega^{\prime}(s) .
\end{aligned}
$$

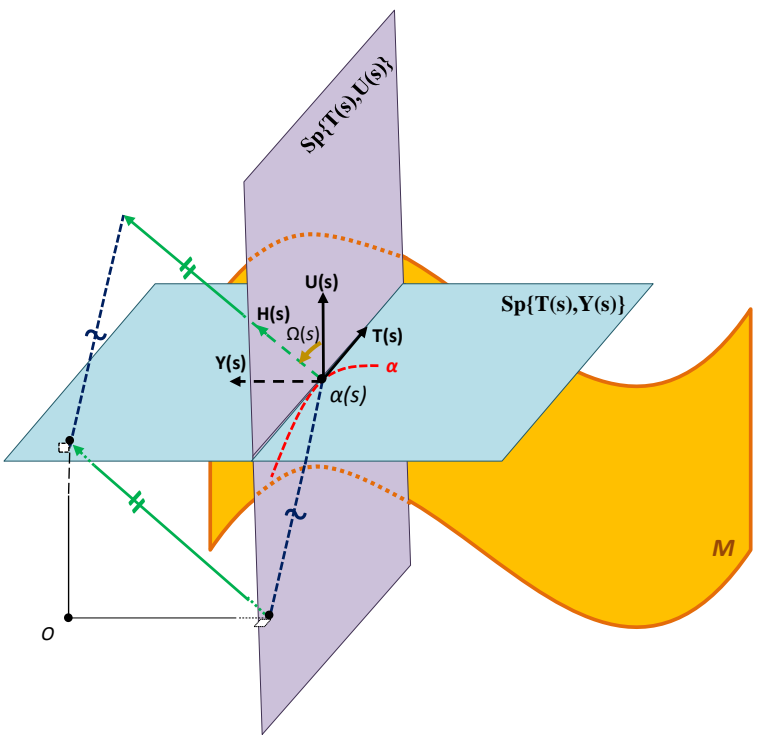

(A)

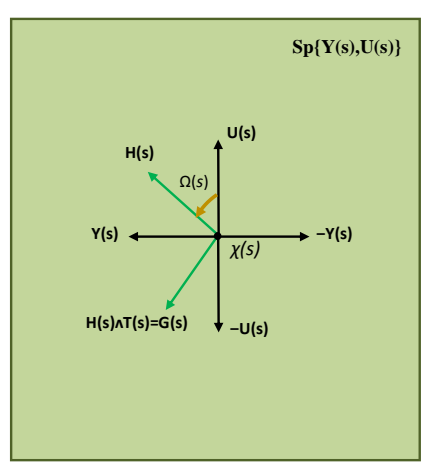

(в)

FIGURE 1. An illustration for PAFORS 
Since

$$
\begin{aligned}
\sin \Omega(s) & =\frac{-\langle\alpha(s), \mathbf{Y}(s)\rangle}{\sqrt{\langle\alpha(s), \mathbf{Y}(s)\rangle^{2}+\langle\alpha(s), \mathbf{U}(s)\rangle^{2}}} \\
\cos \Omega(s) & =\frac{\langle\alpha(s), \mathbf{U}(s)\rangle}{\sqrt{\langle\alpha(s), \mathbf{Y}(s)\rangle^{2}+\langle\alpha(s), \mathbf{U}(s)\rangle^{2}}} \\
\tan \Omega(s) & =-\frac{\langle\alpha(s), \mathbf{Y}(s)\rangle}{\langle\alpha(s), \mathbf{U}(s)\rangle},
\end{aligned}
$$

the rotation angle $\Omega(s)$ is determined as follows:

$$
\Omega(s)=\left\{\begin{array}{c}
\arctan \left(-\frac{\langle\alpha(s), \mathbf{Y}(s)\rangle}{\langle\alpha(s), \mathbf{U}(s)\rangle}\right) \text { if }\langle\alpha(s), \mathbf{U}(s)\rangle>0 \\
\arctan \left(-\frac{\langle\alpha(s), \mathbf{Y}(s)\rangle}{\langle\alpha(s), \mathbf{U}(s)\rangle}\right)+\pi \text { if }\langle\alpha(s), \mathbf{U}(s)\rangle<0 \\
-\frac{\pi}{2} \quad \text { if }\langle\alpha(s), \mathbf{U}(s)\rangle=0, \quad\langle\alpha(s), \mathbf{Y}(s)\rangle>0 \\
\frac{\pi}{2} \text { if }\langle\alpha(s), \mathbf{U}(s)\rangle=0, \quad\langle\alpha(s), \mathbf{Y}(s)\rangle<0 .
\end{array}\right.
$$

Any element of the set $\left\{\mathbf{T}(s), \mathbf{G}(s), \mathbf{H}(s), k_{1}(s), k_{2}(s), k_{3}(s)\right\}$ is called as PAFORS apparatus of the curve $\alpha=\alpha(s)$ [8].

This paper is organized as follows. In Section 2, we consider the geodesic and asymptotic trajectories according to PAFORS in three-dimensional Euclidean space and give some corollaries for the special cases of these trajectories. In Section 3 . we study the slant helical trajectories according to PAFORS and give a method to investigate the existing or not existing of the desired slant helical trajectory on a given implicit surface.

\section{Some Characterizations on Geodesic and Asymptotic Trajectories}

In the remaining sections, we continue to consider any moving point particle on a regular surface $M$ satisfying the aforesaid assumption and to denote the unit speed parameterization of the trajectory by $\alpha=\alpha(s)$. Also, we will show the parameter interval of the trajectory $\alpha=\alpha(s)$ with $I$.

Lemma 2.1. $\alpha=\alpha(s)$ is an asymptotic curve if and only if $k_{1}{ }^{2}+k_{2}{ }^{2}=k_{g}{ }^{2}$.

Proof. From the first and second equations in (1.1),

$$
k_{1}^{2}+k_{2}^{2}={k_{g}}^{2}+k_{n}^{2}
$$

can be easily written. Due to the this equality, the remaining part of the proof is obvious.

Considering above, we give the following lemma without proof.

Lemma 2.2. $\alpha=\alpha(s)$ is a geodesic curve if and only if $k_{1}{ }^{2}+k_{2}{ }^{2}=k_{n}{ }^{2}$.

In the light of the equations (1.1) and 1.2 , the following two remarks can be easily given. 
Remark 1. Let $\alpha=\alpha(s)$ be an asymptotic curve on $M$ with $k_{g} \neq 0$ (The reason why we take $k_{g}$ as nonzero is to avoid $\left.k_{1}=k_{2}=0\right)$. In that case

$$
\begin{aligned}
& k_{1}(s)=k_{g}(s) \frac{\langle\alpha(s), \mathbf{U}(s)\rangle}{\sqrt{\langle\alpha(s), \mathbf{Y}(s)\rangle^{2}+\langle\alpha(s), \mathbf{U}(s)\rangle^{2}}} \\
& k_{2}(s)=-k_{g}(s) \frac{\langle\alpha(s), \mathbf{Y}(s)\rangle}{\sqrt{\langle\alpha(s), \mathbf{Y}(s)\rangle^{2}+\langle\alpha(s), \mathbf{U}(s)\rangle^{2}}}
\end{aligned}
$$

can be immediately written. By keeping $k_{g} \neq 0$ and the assumption concerned with the angular momentum in mind, it is very easy to say that $k_{1}(s)$ and $k_{2}(s)$ do not equal to zero simultaneously and they verify the followings

$$
\begin{aligned}
& k_{1}(s)=0 \quad \Leftrightarrow \quad\langle\alpha(s), \mathbf{U}(s)\rangle=0 \\
& k_{2}(s)=0 \quad \Leftrightarrow \quad\langle\alpha(s), \mathbf{Y}(s)\rangle=0 .
\end{aligned}
$$

As a result of the proposition $k_{1}(s)=0 \Leftrightarrow\langle\alpha(s), \mathbf{U}(s)\rangle=0$, we can write the equality $k_{1}(s)\langle\alpha(s), \mathbf{Y}(s)\rangle+k_{2}(s)\langle\alpha(s), \mathbf{U}(s)\rangle=0$ when $k_{1}(s)=0$. Considering above and using the equations 2.1, (2.2) we have

$$
\frac{k_{2}(s)}{k_{1}(s)}=-\frac{\langle\alpha(s), \mathbf{Y}(s)\rangle}{\langle\alpha(s), \mathbf{U}(s)\rangle}
$$

where $k_{1}(s) \neq 0$. So, we can conclude that

$$
k_{1}(s)\langle\alpha(s), \mathbf{Y}(s)\rangle+k_{2}(s)\langle\alpha(s), \mathbf{U}(s)\rangle=0
$$

is satisfied along the trajectory $\alpha=\alpha(s)$.

Remark 2. Let $\alpha=\alpha(s)$ be a geodesic curve on $M$ with $k_{n} \neq 0$ (The reason why we take $k_{n}$ as nonzero is to avoid $\left.k_{1}=k_{2}=0\right)$. In that case

$$
\begin{aligned}
& k_{1}(s)=k_{n}(s) \frac{\langle\alpha(s), \mathbf{Y}(s)\rangle}{\sqrt{\langle\alpha(s), \mathbf{Y}(s)\rangle^{2}+\langle\alpha(s), \mathbf{U}(s)\rangle^{2}}} \\
& k_{2}(s)=k_{n}(s) \frac{\langle\alpha(s), \mathbf{U}(s)\rangle}{\sqrt{\langle\alpha(s), \mathbf{Y}(s)\rangle^{2}+\langle\alpha(s), \mathbf{U}(s)\rangle^{2}}}
\end{aligned}
$$

can be easily written. Similarly, we can easily say that $k_{1}(s)$ and $k_{2}(s)$ do not equal to zero simultaneously and they verify the followings

$$
\begin{aligned}
& k_{1}(s)=0 \quad \Leftrightarrow \quad\langle\alpha(s), \mathbf{Y}(s)\rangle=0 \\
& k_{2}(s)=0 \quad \Leftrightarrow \quad\langle\alpha(s), \mathbf{U}(s)\rangle=0
\end{aligned}
$$

by keeping the assumption concerned with the angular momentum and $k_{n} \neq 0$ in mind. As a result of the proposition $k_{2}(s)=0 \Leftrightarrow\langle\alpha(s), \mathbf{U}(s)\rangle=0$, we can write $k_{2}(s)\langle\alpha(s), \mathbf{Y}(s)\rangle-k_{1}(s)\langle\alpha(s), \mathbf{U}(s)\rangle=0$ when $k_{2}(s)=0$. Taking into consideration above and using the equations (2.4), 2.5) we have

$$
\frac{k_{1}(s)}{k_{2}(s)}=\frac{\langle\alpha(s), \mathbf{Y}(s)\rangle}{\langle\alpha(s), \mathbf{U}(s)\rangle}
$$

where $k_{2}(s) \neq 0$. Therefore, we can conclude that

$$
k_{2}(s)\langle\alpha(s), \mathbf{Y}(s)\rangle-k_{1}(s)\langle\alpha(s), \mathbf{U}(s)\rangle=0
$$

is satisfied along the trajectory $\alpha=\alpha(s)$. 
Theorem 2.3. Let $\alpha=\alpha(s)$ be an asymptotic curve on $M$ with $k_{g} \neq 0$. In that case, $\alpha=\alpha(s)$ is a curve whose position vector always lies on the instantaneous plane $S p\{\mathbf{T}(s), \mathbf{U}(s)\}$ iff $k_{2}=0$.

Proof. Assume that the asymptotic curve $\alpha$ is a curve whose position vector always lies on the instantaneous plane $\operatorname{Sp}\{\mathbf{T}(s), \mathbf{U}(s)\}$. Then, $\langle\alpha(s), \mathbf{Y}(s)\rangle=0$ for all the values $s$ of parameter. Considering the equation $(2.3)$, we obtain

$$
k_{2}(s)\langle\alpha(s), \mathbf{U}(s)\rangle=0
$$

for all $s$. Because of the non-vanishing angular momentum, $\langle\alpha(s), \mathbf{U}(s)\rangle$ never vanishes along $\alpha$. The first part of the proof is completed by using this information in (2.7).

Conversely, suppose that $k_{2}=0$. From the equation (2.3), we have

$$
\forall s \in I, \quad k_{1}(s)\langle\alpha(s), \mathbf{Y}(s)\rangle=0 .
$$

Due to Remark 1. we know that $k_{1}(s)$ and $k_{2}(s)$ can not equal to zero simultaneously along $\alpha$. Thus, we can conclude that $k_{1}(s)$ never vanishes. This gives us the following

$$
\forall s \in I, \quad\langle\alpha(s), \mathbf{Y}(s)\rangle=0
$$

which means that the asymptotic curve $\alpha$ is a curve whose position vector always lies on the instantaneous plane $S p\{\mathbf{T}(s), \mathbf{U}(s)\}$.

Theorem 2.4. Let $\alpha=\alpha(s)$ be an asymptotic curve on $M$ with $k_{g} \neq 0$. In that case, $\alpha=\alpha(s)$ is a curve whose position vector always lies on the instantaneous plane $\operatorname{Sp}\{\mathbf{T}(s), \mathbf{Y}(s)\}$ iff $k_{1}=0$.

Proof. Assume that the asymptotic curve $\alpha$ is a curve whose position vector always lies on the instantaneous plane $S p\{\mathbf{T}(s), \mathbf{Y}(s)\}$. In this case, $\langle\alpha(s), \mathbf{U}(s)\rangle=0$ for all the values $s$ of the arc-length parameter. Taking into account of the equation 2.3 , we have

$$
k_{1}(s)\langle\alpha(s), \mathbf{Y}(s)\rangle=0
$$

for all $s$. Similarly above, $\langle\alpha(s), \mathbf{Y}(s)\rangle$ never vanishes along $\alpha$ thanks to the nonvanishing angular momentum. Then

$$
\forall s \in I, \quad k_{1}(s)=0
$$

can be concluded and this finishes the first part of the proof.

Conversely, assume that $k_{1}=0$. In that case, from the equation 2.3

$$
\forall s \in I, \quad k_{2}(s)\langle\alpha(s), \mathbf{U}(s)\rangle=0
$$

can be written. Because $k_{1}(s)$ and $k_{2}(s)$ do not equal to zero simultaneously along $\alpha=\alpha(s)$, we can say

$$
\forall s \in I, \quad k_{2}(s) \neq 0 .
$$

This yields the following

$$
\forall s \in I, \quad\langle\alpha(s), \mathbf{U}(s)\rangle=0
$$

which means that the asymptotic curve $\alpha$ is a curve whose position vector always lies on the instantaneous plane $S p\{\mathbf{T}(s), \mathbf{Y}(s)\}$.

Theorem 2.5. Let $\alpha=\alpha(s)$ be an asymptotic curve on $M$ with $k_{g} \neq 0$. Then the following properties hold: 
(1) If the position vector of $\alpha=\alpha(s)$ always lies on the instantaneous plane $\operatorname{Sp}\{\mathbf{Y}(s), \mathbf{U}(s)\}$, differential equation $k_{1}{k_{2}}^{\prime}-k_{2} k_{1}{ }^{\prime}+\left(\Omega^{\prime}+k_{3}\right)\left(k_{1}{ }^{2}+k_{2}{ }^{2}\right)=0$ is satisfied along $\alpha$.

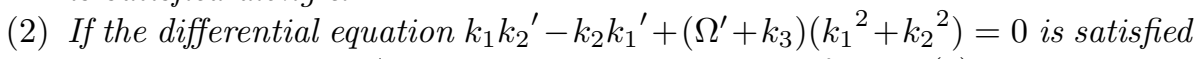
along $\alpha$ with $k_{1}, k_{2} \neq 0$, then the position vector of $\alpha=\alpha(s)$ always lies on the instantaneous plane $\operatorname{Sp}\{\mathbf{Y}(s), \mathbf{U}(s)\}$.

Proof. One can easily find the equation

$$
\begin{aligned}
0= & -\left(k_{1}{ }^{2}(s)+k_{2}{ }^{2}(s)\right) \cos \Omega(s)\langle\alpha(s), \mathbf{T}(s)\rangle \\
& +\left(-k_{2}(s)\left(k_{3}(s)+\Omega^{\prime}(s)\right)+k_{1}{ }^{\prime}(s)\right)\langle\alpha(s), \mathbf{Y}(s)\rangle \\
& +\left(k_{1}(s)\left(k_{3}(s)+\Omega^{\prime}(s)\right)+k_{2}{ }^{\prime}(s)\right)\langle\alpha(s), \mathbf{U}(s)\rangle
\end{aligned}
$$

by differentiating (2.3) and considering the relations between the PAFORS apparatus and Darboux apparatus. If the necessary operations are applied to the equations 2.3 and 2.8 side by side,

$$
\begin{aligned}
0= & k_{2}(s)\left(k_{1}{ }^{2}(s)+{k_{2}}^{2}(s)\right) \cos \Omega(s)\langle\alpha(s), \mathbf{T}(s)\rangle \\
& +\left(k_{1}(s) k_{2}{ }^{\prime}(s)-k_{2}(s) k_{1}{ }^{\prime}(s)+\left(\Omega^{\prime}(s)+k_{3}(s)\right)\left(k_{1}{ }^{2}(s)+k_{2}{ }^{2}(s)\right)\right)\langle\alpha(s), \mathbf{Y}(s)\rangle
\end{aligned}
$$

can be obtained. Now we can investigate the items:

(1) Assume that the position vector of $\alpha=\alpha(s)$ always lies on the instantaneous plane $S p\{\mathbf{Y}(s), \mathbf{U}(s)\}$. Then,

$$
\langle\alpha(s), \mathbf{T}(s)\rangle=0
$$

for all the values $s$. Taking into consideration 2.9 , we get

$$
\left(k_{1}(s) k_{2}{ }^{\prime}(s)-k_{2}(s) k_{1}{ }^{\prime}(s)+\left(\Omega^{\prime}(s)+k_{3}(s)\right)\left(k_{1}{ }^{2}(s)+k_{2}{ }^{2}(s)\right)\right)\langle\alpha(s), \mathbf{Y}(s)\rangle=0
$$

for all $s$. Also, differentiating the equation 2.10 gives us the following:

$$
k_{g}(s)\langle\alpha(s), \mathbf{Y}(s)\rangle+k_{n}(s)\langle\alpha(s), \mathbf{U}(s)\rangle=-1 .
$$

Since $k_{n}=0$ for the asymptotic curve $\alpha=\alpha(s)$,

$$
\forall s \in I, \quad\langle\alpha(s), \mathbf{Y}(s)\rangle=-\frac{1}{k_{g}(s)} \neq 0
$$

can be written. Therefore,

$$
k_{1} k_{2}{ }^{\prime}-k_{2} k_{1}{ }^{\prime}+\left(\Omega^{\prime}+k_{3}\right)\left(k_{1}{ }^{2}+{k_{2}}^{2}\right)=0
$$

is obtained from 2.11 and 2.12.

(2) Let the differential equation $k_{1} k_{2}{ }^{\prime}-k_{2} k_{1}{ }^{\prime}+\left(\Omega^{\prime}+k_{3}\right)\left(k_{1}{ }^{2}+k_{2}{ }^{2}\right)=0$ be satisfied along $\alpha$ with $k_{1}, k_{2} \neq 0$. In the light of $(2.9)$, we can write

$$
\forall s \in I, \quad k_{2}(s)\left(k_{1}^{2}(s)+k_{2}^{2}(s)\right) \cos \Omega(s)\langle\alpha(s), \mathbf{T}(s)\rangle=0 .
$$

Because $k_{1}, k_{2} \neq 0$, we get

$$
\forall s \in I, \quad\langle\alpha(s), \mathbf{T}(s)\rangle=0
$$

which means that the position vector of the asymptotic curve $\alpha$ always lies on the instantaneous plane $S p\{\mathbf{Y}(s), \mathbf{U}(s)\}$ (Notice that $\cos \Omega(s)=\frac{k_{1}(s)}{k_{g}(s)}$ under these conditions). 
Theorem 2.6. Let $\alpha=\alpha(s)$ be a geodesic curve on $M$ with $k_{n} \neq 0$. In that case, $\alpha=\alpha(s)$ is a curve whose position vector always lies on the instantaneous plane $S p\{\mathbf{T}(s), \mathbf{U}(s)\}$ iff $k_{1}=0$.

Proof. Assume that the geodesic curve $\alpha$ is a curve whose position vector always lies on the instantaneous plane $S p\{\mathbf{T}(s), \mathbf{U}(s)\}$. Then, $\langle\alpha(s), \mathbf{Y}(s)\rangle=0$ for all the values $s$. Taking into account of $(2.6)$, we get

$$
-k_{1}(s)\langle\alpha(s), \mathbf{U}(s)\rangle=0
$$

for all $s$. Because of the non-vanishing angular momentum, $\langle\alpha(s), \mathbf{U}(s)\rangle$ never vanishes along $\alpha$. Substituting this into 2.13 completes the first part of the proof.

Conversely, assume that $k_{1}=0$. From (2.6), we obtain

$$
\forall s \in I, \quad k_{2}(s)\langle\alpha(s), \mathbf{Y}(s)\rangle=0 .
$$

Due to Remark 2, we know that $k_{1}(s)$ and $k_{2}(s)$ can not equal to zero simultaneously along $\alpha$. Thus, we can conclude that $k_{2}(s)$ never vanishes. This yields

$$
\forall s \in I, \quad\langle\alpha(s), \mathbf{Y}(s)\rangle=0
$$

which means that the geodesic curve $\alpha$ is a curve whose position vector always lies on the instantaneous plane $S p\{\mathbf{T}(s), \mathbf{U}(s)\}$.

Theorem 2.7. Let $\alpha=\alpha(s)$ be a geodesic curve on $M$ with $k_{n} \neq 0$. In that case, $\alpha=\alpha(s)$ is a curve whose position vector always lies on the instantaneous plane $S p\{\mathbf{T}(s), \mathbf{Y}(s)\}$ iff $k_{2}=0$.

Proof. Suppose that the geodesic curve $\alpha$ is a curve whose position vector always lies on the instantaneous plane $S p\{\mathbf{T}(s), \mathbf{Y}(s)\}$. Then, $\langle\alpha(s), \mathbf{U}(s)\rangle=0$ for all the values $s$. Considering (2.6), we find

$$
k_{2}(s)\langle\alpha(s), \mathbf{Y}(s)\rangle=0
$$

for all $s$. Similarly previous proof, we can say that $\langle\alpha(s), \mathbf{Y}(s)\rangle$ never vanishes along $\alpha$ in the light of non-vanishing angular momentum. Then

$$
\forall s \in I, \quad k_{2}(s)=0
$$

can be concluded.

Conversely, assume that $k_{2}=0$. In that case, from 2.6

$$
\forall s \in I, \quad-k_{1}(s)\langle\alpha(s), \mathbf{U}(s)\rangle=0
$$

can be written. Because $k_{1}(s)$ and $k_{2}(s)$ do not equal to zero simultaneously along $\alpha$, we find

$$
\forall s \in I, \quad k_{1}(s) \neq 0 .
$$

This yields the following

$$
\forall s \in I, \quad\langle\alpha(s), \mathbf{U}(s)\rangle=0
$$

which means that the geodesic curve $\alpha$ is a curve whose position vector always lies on the instantaneous plane $S p\{\mathbf{T}(s), \mathbf{Y}(s)\}$.

Theorem 2.8. Let $\alpha=\alpha(s)$ be a geodesic curve on $M$ with $k_{n} \neq 0$. Then the following properties hold:

(1) If the position vector of $\alpha=\alpha(s)$ always lies on the instantaneous plane $S p\{\mathbf{Y}(s), \mathbf{U}(s)\}$, differential equation $k_{2} k_{1}{ }^{\prime}-k_{1} k_{2}{ }^{\prime}-\left(k_{3}+\Omega^{\prime}\right)\left(k_{1}{ }^{2}+k_{2}{ }^{2}\right)=0$ is satisfied along $\alpha$. 
(2) If the differential equation $k_{2} k_{1}{ }^{\prime}-k_{1} k_{2}{ }^{\prime}-\left(k_{3}+\Omega^{\prime}\right)\left(k_{1}{ }^{2}+k_{2}{ }^{2}\right)=0$ is satisfied along $\alpha$ with $k_{1}, k_{2} \neq 0$, then the position vector of $\alpha=\alpha(s)$ always lies on the instantaneous plane $\operatorname{Sp}\{\mathbf{Y}(s), \mathbf{U}(s)\}$.

Proof. One can easily find the equation

$$
\begin{aligned}
0= & -\left(k_{1}{ }^{2}(s)+{k_{2}}^{2}(s)\right) \sin \Omega(s)\langle\alpha(s), \mathbf{T}(s)\rangle \\
& +\left(k_{1}(s)\left(k_{3}(s)+\Omega^{\prime}(s)\right)+k_{2}{ }^{\prime}(s)\right)\langle\alpha(s), \mathbf{Y}(s)\rangle \\
& +\left(k_{2}(s)\left(k_{3}(s)+\Omega^{\prime}(s)\right)-k_{1}{ }^{\prime}(s)\right)\langle\alpha(s), \mathbf{U}(s)\rangle
\end{aligned}
$$

by differentiating (2.6) and taking into consideration the relations between the PAFORS apparatus and Darboux apparatus. If the necessary operations are applied to the equations (2.6) and (2.14) side by side, the equation

$$
\begin{aligned}
0= & k_{2}(s)\left(k_{1}{ }^{2}(s)+k_{2}{ }^{2}(s)\right) \sin \Omega(s)\langle\alpha(s), \mathbf{T}(s)\rangle \\
& +\left(k_{2}(s) k_{1}{ }^{\prime}(s)-k_{1}(s) k_{2}{ }^{\prime}(s)-\left(k_{3}(s)+\Omega^{\prime}(s)\right)\left(k_{1}{ }^{2}(s)+k_{2}{ }^{2}(s)\right)\right)\langle\alpha(s), \mathbf{U}(s)\rangle
\end{aligned}
$$

can be obtained. Now we can investigate the items:

(1) Assume that the position vector of $\alpha=\alpha(s)$ always lies on the instantaneous plane $S p\{\mathbf{Y}(s), \mathbf{U}(s)\}$. Then,

$$
\langle\alpha(s), \mathbf{T}(s)\rangle=0
$$

for all the values $s$. Considering the equation 2.15), we get

$$
\left(k_{2}(s) k_{1}{ }^{\prime}(s)-k_{1}(s) k_{2}{ }^{\prime}(s)-\left(k_{3}(s)+\Omega^{\prime}(s)\right)\left(k_{1}{ }^{2}(s)+k_{2}{ }^{2}(s)\right)\right)\langle\alpha(s), \mathbf{U}(s)\rangle=0
$$

for all $s$. Also, differentiating (2.16) yields the equation

$$
k_{g}(s)\langle\alpha(s), \mathbf{Y}(s)\rangle+k_{n}(s)\langle\alpha(s), \mathbf{U}(s)\rangle=-1 .
$$

Because $k_{g}=0$ for the geodesic curve $\alpha=\alpha(s)$,

$$
\forall s \in I, \quad\langle\alpha(s), \mathbf{U}(s)\rangle=-\frac{1}{k_{n}(s)} \neq 0
$$

can be written. Thus,

$$
k_{2} k_{1}{ }^{\prime}-k_{1} k_{2}{ }^{\prime}-\left(k_{3}+\Omega^{\prime}\right)\left(k_{1}{ }^{2}+k_{2}{ }^{2}\right)=0
$$

is obtained from 2.17) and 2.18).

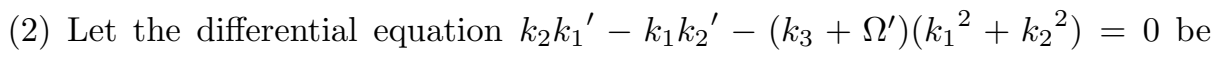
satisfied along $\alpha$ with $k_{1}, k_{2} \neq 0$. In the light of 2.15 , we can write

$$
\forall s \in I, \quad k_{2}(s)\left(k_{1}{ }^{2}(s)+k_{2}{ }^{2}(s)\right) \sin \Omega(s)\langle\alpha(s), \mathbf{T}(s)\rangle=0 .
$$

Because $k_{1}, k_{2} \neq 0$, we find

$$
\forall s \in I, \quad\langle\alpha(s), \mathbf{T}(s)\rangle=0
$$

which means that the position vector of the geodesic curve $\alpha$ always lies on the instantaneous plane $S p\{\mathbf{Y}(s), \mathbf{U}(s)\}$ (Notice that $\sin \Omega(s)=-\frac{k_{1}(s)}{k_{n}(s)}$ under these conditions). 


\section{Slant Helical Trajectories According to PAFORS}

Slant helix was expressed in [10] as a curve whose principal normal vector makes a constant angle with a fixed direction in $E^{3}$. Until now, several kinds of slant helices have been defined and studied in the literature. There can be found some of them in $11-14$. In this section, we take into consideration the slant helical trajectories according to PAFORS and discuss some special cases of them. Also, we give a method to investigate the existing or not existing of the desired slant helical trajectory on a given implicit surface. Note that similar steps and approaches in 15 and [16] will be followed in this section.

As mentioned earlier, we continue to consider any moving point particle on a regular surface $M$ satisfying the aforesaid assumption and to denote the unit speed parameterization of the trajectory by $\alpha=\alpha(s)$.

Firstly, we define $\mathbf{G}$-PAFORS spherical image of the trajectory. We consider this spherical image since it has an important place for the characterization of our slant helical trajectories. The remaining PAFORS spherical images can be topic of a different study.

Definition 3.1. If we move the vector field $\mathbf{G}$ of $\alpha=\alpha(s)$ to the center $O$ of the unit sphere $S^{2}$, we find a curve which $\mathbf{G}(s)$ draws on $S^{2}$. We call this curve $\mathbf{G}-$ PAFORS spherical image of $\alpha=\alpha(s)$ and show it with $\xi_{G}$.

For $\mathbf{G}-\mathrm{PAFORS}$ spherical image of $\alpha=\alpha(s)$, we can write

$$
\xi_{G}(s)=\mathbf{G}(s) .
$$

If this equation is differentiated with respect to $s$, we get

$$
\begin{aligned}
\xi_{G}^{\prime}(s)= & -k_{1}(s) \mathbf{T}(s)+k_{3}(s) \mathbf{H}(s) \\
\xi_{G}^{\prime \prime}(s)= & {\left[-k_{1}{ }^{\prime}(s)-k_{2}(s) k_{3}(s)\right] \mathbf{T}(s)-\left[k_{1}{ }^{2}(s)+k_{3}{ }^{2}(s)\right] \mathbf{G}(s) } \\
& +\left[-k_{1}(s) k_{2}(s)+k_{3}{ }^{\prime}(s)\right] \mathbf{H}(s) \\
\xi^{\prime \prime \prime}{ }_{G}(s)= & {\left[-k_{1}{ }^{\prime \prime}(s)-k_{2}{ }^{\prime}(s) k_{3}(s)-2 k_{3}{ }^{\prime}(s) k_{2}(s)+k_{1}(s)\left(k_{1}{ }^{2}(s)+k_{2}{ }^{2}(s)+k_{3}{ }^{2}(s)\right)\right] \mathbf{T}(s) } \\
& -3\left[k_{1}(s) k_{1}{ }^{\prime}(s)+k_{3}(s) k_{3}{ }^{\prime}(s)\right] \mathbf{G}(s) \\
& +\left[k_{3}{ }^{\prime \prime}(s)-k_{2}{ }^{\prime}(s) k_{1}(s)-2 k_{1}{ }^{\prime}(s) k_{2}(s)-k_{3}(s)\left(k_{1}{ }^{2}(s)+k_{2}{ }^{2}(s)+k_{3}{ }^{2}(s)\right)\right] \mathbf{H}(s) .
\end{aligned}
$$

These equations yield the curvature $\kappa_{G}$ and the torsion $\tau_{G}$ of $\xi_{G}$ as in the following:

$$
\begin{aligned}
\kappa_{G}(s) & =\frac{\left\|\xi_{G}^{\prime}(s) \wedge \xi_{G}^{\prime \prime}(s)\right\|}{\left\|\xi_{G}^{\prime}(s)\right\|^{3}}=\sqrt{1+\left(\zeta_{G}(s)\right)^{2}} \\
\tau_{G}(s) & =\frac{\left\langle\xi_{G}^{\prime}(s) \wedge \xi^{\prime \prime}{ }_{G}(s), \xi^{\prime \prime \prime}{ }_{G}(s)\right\rangle}{\left\|\xi_{G}^{\prime}(s) \wedge \xi^{\prime \prime}{ }_{G}(s)\right\|^{2}}=\frac{\zeta_{G}^{\prime}(s)}{\left(1+\left(\zeta_{G}(s)\right)^{2}\right)\left(k_{1}{ }^{2}(s)+k_{3}{ }^{2}(s)\right)^{11 / 2}}
\end{aligned}
$$

where

$$
\zeta_{G}(s)=\left(\frac{k_{3}{ }^{\prime} k_{1}-k_{1}{ }^{\prime} k_{3}-k_{2}\left(k_{1}{ }^{2}+k_{3}{ }^{2}\right)}{\left(k_{1}{ }^{2}+k_{3}{ }^{2}\right)^{3 / 2}}\right)(s) .
$$

Definition 3.2. $\alpha=\alpha(s)$ is called a slant helical trajectory (according to PAFORS) if the vector field $\mathbf{G}$ of $\alpha=\alpha(s)$ makes a constant angle with a fixed direction.

If $\alpha=\alpha(s)$ is a slant helical trajectory according to PAFORS, then there exist a constant angle $\beta$ and a fixed unit vector $\mathbf{g}$ which satisfy

$$
\langle\mathbf{G}(s), \mathbf{g}\rangle=\cos \beta
$$

for all $s$. 
Theorem 3.1. Assume that $\left(k_{1}(s), k_{3}(s)\right) \neq(0,0)$. Then, $\alpha$ is a slant helical trajectory according to PAFORS iff the function in (3.3) is a constant function.

Proof. Let $\alpha=\alpha(s)$ with $\left(k_{1}(s), k_{3}(s)\right) \neq(0,0)$ be slant helical trajectory according to PAFORS in $E^{3}$. In this case, from Definition 3.2. $\mathbf{G}$ makes a constant angle with a fixed direction. Therefore, $\mathbf{G}$-PAFORS spherical image $\xi_{G}$ of $\alpha=\alpha(s)$ is part of a circle. Thus, it has constant curvature and zero torsion. Using this information in 3.1 and 3.2 , we can immediately conclude $\zeta_{G}(s)=$ constant.

Conversely, suppose that $\zeta_{G}(s)=$ constant. In this case, it is not difficult to see that $\kappa_{G}(s)=$ constant and $\tau_{G}=0$. Therefore, $\mathbf{G}$-PAFORS spherical image $\xi_{G}$ of $\alpha=\alpha(s)$ is part of a circle. So, $\mathbf{G}$ makes a constant angle with a fixed direction and we can finish the proof.

Corollary 3.2. Let $\alpha=\alpha(s)$ be an asymptotic curve on $M$ with $k_{g}, k_{1} \neq 0$. Assume that its position vector always lies on the instantaneous plane $S p\{\mathbf{T}(s), \mathbf{U}(s)\}$. Then, $\alpha$ is a slant helical trajectory according to PAFORS iff

$$
\left(\frac{k_{1}{ }^{2}}{\left(k_{1}{ }^{2}+k_{3}{ }^{2}\right)^{3 / 2}}\left(\frac{k_{3}}{k_{1}}\right)^{\prime}\right)(s)
$$

is a constant function.

Proof. Where $k_{g}, k_{1} \neq 0$, let the asymptotic curve $\alpha=\alpha(s)$, whose position vector always lies on the instantaneous plane $S p\{\mathbf{T}(s), \mathbf{U}(s)\}$, be a slant helical trajectory according to PAFORS. From Theorem 2.3. we can write $k_{2}=0$. If we use this in 3.3 , we get

$$
\zeta_{G}(s)=\left(\frac{k_{1}^{2}}{\left(k_{1}^{2}+{k_{3}}^{2}\right)^{3 / 2}}\left(\frac{k_{3}}{k_{1}}\right)^{\prime}\right)(s) .
$$

In that case, Theorem 3.1 finishes the first part of the proof. Similarly, one can easily complete the other part of the proof.

Corollary 3.3. Let $\alpha=\alpha(s)$ be an asymptotic curve on $M$ with non-zero $k_{g}$ and non-zero $k_{3}$. Assume that its position vector always lies on the instantaneous plane $S p\{\mathbf{T}(s), \mathbf{Y}(s)\}$. Then, $\alpha$ is a slant helical trajectory according to PAFORS iff

$$
\left(\frac{k_{2}}{k_{3}}\right)(s)
$$

is a constant function.

Proof. Where $k_{g}, k_{3} \neq 0$, let the asymptotic curve $\alpha=\alpha(s)$, whose position vector always lies on the instantaneous plane $S p\{\mathbf{T}(s), \mathbf{Y}(s)\}$, be a slant helical trajectory according to PAFORS. From Theorem 2.4, we can write $k_{1}=0$. If we use this in (3.3), we find

$$
\zeta_{G}(s)=\left(-\frac{k_{2}}{k_{3}}\right)(s)
$$

In this case, Theorem 3.1 finishes the first part of the proof. Similarly, the other part of the proof is easily completed. So, we omit it. 
Corollary 3.4. Let $\alpha=\alpha(s)$ be a geodesic curve on $M$ with non-zero $k_{n}$ and non-zero $k_{1}$. Assume that its position vector always lies on the instantaneous plane $S p\{\mathbf{T}(s), \mathbf{Y}(s)\}$. Then, $\alpha$ is a slant helical trajectory according to PAFORS iff

$$
\left(\frac{k_{1}{ }^{2}}{\left(k_{1}{ }^{2}+k_{3}{ }^{2}\right)^{3 / 2}}\left(\frac{k_{3}}{k_{1}}\right)^{\prime}\right)(s)
$$

is a constant function.

Proof. Where $k_{n}, k_{1} \neq 0$, let the geodesic curve $\alpha=\alpha(s)$, whose position vector always lies on the instantaneous plane $S p\{\mathbf{T}(s), \mathbf{Y}(s)\}$, be a slant helical trajectory according to PAFORS. From Theorem 2.7, we can write $k_{2}=0$. If $k_{2}=0$ is substituted in (3.3), we find

$$
\zeta_{G}(s)=\left(\frac{k_{1}^{2}}{\left(k_{1}{ }^{2}+{k_{3}}^{2}\right)^{3 / 2}}\left(\frac{k_{3}}{k_{1}}\right)^{\prime}\right)(s) .
$$

Then, the first part of the proof is easily finished considering Theorem 3.1. Similarly, one can easily complete the other part of the proof.

Corollary 3.5. Let $\alpha=\alpha(s)$ be a geodesic curve on $M$ with non-zero $k_{n}$ and non-zero $k_{3}$. Assume that its position vector always lies on the instantaneous plane $S p\{\mathbf{T}(s), \mathbf{U}(s)\}$. Then, $\alpha$ is a slant helical trajectory according to PAFORS iff

$$
\left(\frac{k_{2}}{k_{3}}\right)(s)
$$

is a constant function.

Proof. Where $k_{n}, k_{3} \neq 0$, let the geodesic curve $\alpha=\alpha(s)$, whose position vector always lies on the instantaneous plane $S p\{\mathbf{T}(s), \mathbf{U}(s)\}$, be a slant helical trajectory according to PAFORS. From Theorem 2.6, we can write $k_{1}=0$. If $k_{1}=0$ is substituted in (3.3), we obtain

$$
\zeta_{G}(s)=\left(-\frac{k_{2}}{k_{3}}\right)(s)
$$

In that case, Theorem 3.1 finishes the first part of the proof. Similarly, the other part of the proof is immediately completed. So, we omit it.

\subsection{Determination of the helix axis for slant helical trajectories.}

In this subsection, we will discuss on the determination of the helix axis for the slant helical trajectories. Let $\alpha=\alpha(s)$ be a slant helical trajectory according to PAFORS. In that case, there exist a constant angle $\beta$ and a fixed unit vector $\mathbf{g}$ which satisfy $\langle\mathbf{G}, \mathbf{g}\rangle=\cos \beta=\lambda_{2}$ where $\mathbf{g}=\lambda_{1} \mathbf{T}+\lambda_{2} \mathbf{G}+\lambda_{3} \mathbf{H}$. With the aid of differentiation with respect to $s$, we obtain

$$
\left\langle-k_{1} \mathbf{T}+k_{3} \mathbf{H}, \mathbf{g}\right\rangle=0 .
$$

Now, let us differentiate the vector $\mathbf{g}$. In that case,

$$
\left(\lambda_{1}{ }^{\prime}-\lambda_{2} k_{1}-\lambda_{3} k_{2}\right) \mathbf{T}+\left(\lambda_{1} k_{1}-\lambda_{3} k_{3}\right) \mathbf{G}+\left(\lambda_{3}{ }^{\prime}+\lambda_{1} k_{2}+\lambda_{2} k_{3}\right) \mathbf{H}=0
$$

is found. This gives us the equation system

$$
\begin{aligned}
\lambda_{1}{ }^{\prime}-\lambda_{2} k_{1}-\lambda_{3} k_{2} & =0 \\
\lambda_{1} k_{1}-\lambda_{3} k_{3} & =0 \\
\lambda_{3}{ }^{\prime}+\lambda_{1} k_{2}+\lambda_{2} k_{3} & =0 .
\end{aligned}
$$


Let us solve this system. Firstly, we must emphasize that we will follow similar steps given in [15 and 16 to find the solution of this system. If the equality $\lambda_{1}=\frac{k_{3}}{k_{1}} \lambda_{3}\left(k_{1}(s) \neq 0\right)$ is written in the equations $\left.3.5 n_{1}, 3.5\right)_{3}$ and some necessary operations are applied to these two new equations, we find the differential equation

$$
\left(1+\left(\frac{k_{3}}{k_{1}}\right)^{2}\right) \lambda_{3}{ }^{\prime}+\frac{k_{3}}{k_{1}}\left(\frac{k_{3}}{k_{1}}\right)^{\prime} \lambda_{3}=0 .
$$

General solution of this equation can be easily obtained as $\lambda_{3}=\mu \frac{k_{1}}{\sqrt{k_{1}^{2}+k_{3}^{2}}}$ where $\mu$ is the constant of integration. In that case, it is very easy to find $\lambda_{1}=\mu \frac{k_{3}}{\sqrt{k_{1}^{2}+k_{3}{ }^{2}}}$ from the relation $\lambda_{1}=\frac{k_{3}}{k_{1}} \lambda_{3}$. Because the vector $\mathbf{g}=\mu \frac{k_{3}}{\sqrt{k_{1}{ }^{2}+k_{3}{ }^{2}}} \mathbf{T}+\cos \beta \mathbf{G}+$ $\mu \frac{k_{1}}{\sqrt{k_{1}^{2}+k_{3}^{2}}} \mathbf{H}$ is taken as a unit vector, the integration constant is derived as $\mu=$ $\pm \sin \beta$. Therefore, $\mathbf{g}= \pm \frac{k_{3}}{\sqrt{k_{1}^{2}+k_{3}^{2}}} \sin \beta \mathbf{T}+\cos \beta \mathbf{G} \pm \frac{k_{1}}{\sqrt{k_{1}^{2}+k_{3}{ }^{2}}} \sin \beta \mathbf{H}$ can be immediately written. Finally, the constant angle $\beta$ must be determined. Differentiating (3.4) with respect to $s$,

$$
\left\langle\left(-k_{1}{ }^{\prime}-k_{2} k_{3}\right) \mathbf{T}+\left(-k_{1}{ }^{2}-k_{3}{ }^{2}\right) \mathbf{G}+\left(k_{3}{ }^{\prime}-k_{1} k_{2}\right) \mathbf{H}, \mathbf{g}\right\rangle=0
$$

is found. Thus, we get

$$
\pm \sin \beta\left(\frac{k_{1} k_{3}{ }^{\prime}-k_{3} k_{1}{ }^{\prime}-k_{2} k_{1}{ }^{2}-k_{2} k_{3}{ }^{2}}{\sqrt{k_{1}{ }^{2}+k_{3}{ }^{2}}}\right)-\cos \beta\left(k_{1}{ }^{2}+k_{3}{ }^{2}\right)=0 .
$$

This gives us the following:

$$
\tan \beta= \pm \frac{\left(k_{1}{ }^{2}+k_{3}{ }^{2}\right)^{3 / 2}}{k_{1} k_{3}{ }^{\prime}-k_{3} k_{1}{ }^{\prime}-k_{2} k_{1}{ }^{2}-k_{2} k_{3}{ }^{2}} .
$$

By means of the above information, one can easily find $\beta$ and determine the fixed direction generated by the constant vector $\mathbf{g}$ for the slant helical trajectory according to PAFORS.

\subsection{Calculating a slant helical trajectory on a given implicit surface.}

In Euclidean 3-space, suppose that the regular surface $M$ is given in implicit form by $f\left(x_{1}, x_{2}, x_{3}\right)=0$. Let us try to generate a slant helical trajectory lying on $M$. That is, our aim is to give a method which enables us to find the slant helical trajectory $\alpha(s)=\left(x_{1}(s), x_{2}(s), x_{3}(s)\right)$ according to PAFORS (if exists) lying on $M$ which accepts a given fixed unit direction $\mathbf{g}=(a, b, c)$ as an axis and a given angle $\beta$ as the constant angle (Note that $s$ is the arc-length parameter of $\alpha$ ). As it is clear that the unit speed curve $\alpha(s)=\left(x_{1}(s), x_{2}(s), x_{3}(s)\right)$ on $M$ satisfies

$$
\left\langle\nabla f, \frac{d \alpha}{d s}\right\rangle=f_{x_{1}} \frac{d x_{1}}{d s}+f_{x_{2}} \frac{d x_{2}}{d s}+f_{x_{3}} \frac{d x_{3}}{d s}=0
$$

and

$$
\left\|\frac{d \alpha}{d s}\right\|=\left(\frac{d x_{1}}{d s}\right)^{2}+\left(\frac{d x_{2}}{d s}\right)^{2}+\left(\frac{d x_{3}}{d s}\right)^{2}=1
$$

where $\nabla f=\left(\frac{\partial f}{\partial x_{1}}, \frac{\partial f}{\partial x_{2}}, \frac{\partial f}{\partial x_{3}}\right)=\left(f_{x_{1}}, f_{x_{2}}, f_{x_{3}}\right)$ and $\frac{d \alpha}{d s}=\left(\frac{d x_{1}}{d s}, \frac{d x_{2}}{d s}, \frac{d x_{3}}{d s}\right)$. We want to obtain $x_{1}(s), x_{2}(s)$ and $x_{3}(s)$ to determine $\alpha(s)$. 
Let $(\mathbf{T}, \mathbf{G}, \mathbf{H})$ and $(\mathbf{T}, \mathbf{Y}, \mathbf{U})$ denote PAFORS and Darboux frame for the trajectory $\alpha(s)=\left(x_{1}(s), x_{2}(s), x_{3}(s)\right)$, respectively. From the theory of curves and surfaces we know that $\mathbf{U}=\frac{\nabla f}{\|\nabla f\|}$ and $\mathbf{Y}=\frac{\nabla f}{\|\nabla f\|} \wedge \mathbf{T}$ can be written easily. In view of the relation of PAFORS and Darboux frame, we get

$$
\begin{aligned}
\mathbf{G}= & \cos \Omega \mathbf{Y}-\sin \Omega \mathbf{U} \\
= & \cos \Omega\left(\frac{\nabla f}{\|\nabla f\|} \wedge \mathbf{T}\right)-\sin \Omega \frac{\nabla f}{\|\nabla f\|} \\
= & \frac{1}{\|\nabla f\|} \cos \Omega(\nabla f \wedge \mathbf{T})-\frac{1}{\|\nabla f\|} \sin \Omega \nabla f \\
= & \frac{1}{\|\nabla f\|} \cos \Omega\left(f_{x_{2}} \frac{d x_{3}}{d s}-f_{x_{3}} \frac{d x_{2}}{d s}, f_{x_{3}} \frac{d x_{1}}{d s}-f_{x_{1}} \frac{d x_{3}}{d s}, f_{x_{1}} \frac{d x_{2}}{d s}-f_{x_{2}} \frac{d x_{1}}{d s}\right) \\
& -\frac{1}{\|\nabla f\|} \sin \Omega\left(f_{x_{1}}, f_{x_{2}}, f_{x_{3}}\right) \\
= & \frac{1}{\|\nabla f\|}\left(\begin{array}{l}
f_{x_{2}} \cos \Omega \frac{d x_{3}}{d s}-f_{x_{3}} \cos \Omega \frac{d x_{2}}{d s}-f_{x_{1}} \sin \Omega, \\
f_{x_{3}} \cos \Omega \frac{d x_{1}}{d s}-f_{x_{1}} \cos \Omega \frac{d x_{3}}{d s}-f_{x_{2}} \sin \Omega, \\
f_{x_{1}} \cos \Omega \frac{d x_{2}}{d s}-f_{x_{2}} \cos \Omega \frac{d x_{1}}{d s}-f_{x_{3}} \sin \Omega
\end{array}\right) .
\end{aligned}
$$

If this last equation is considered in $\langle\mathbf{G}, \mathbf{g}\rangle=\cos \beta$,

$$
\begin{aligned}
\|\nabla f\| \cos \beta= & \left(a f_{x_{2}} \cos \Omega \frac{d x_{3}}{d s}-a f_{x_{3}} \cos \Omega \frac{d x_{2}}{d s}-a f_{x_{1}} \sin \Omega\right) \\
& +\left(b f_{x_{3}} \cos \Omega \frac{d x_{1}}{d s}-b f_{x_{1}} \cos \Omega \frac{d x_{3}}{d s}-b f_{x_{2}} \sin \Omega\right) \\
& +\left(c f_{x_{1}} \cos \Omega \frac{d x_{2}}{d s}-c f_{x_{2}} \cos \Omega \frac{d x_{1}}{d s}-c f_{x_{3}} \sin \Omega\right)
\end{aligned}
$$

and

$$
\begin{aligned}
\|\nabla f\| \cos \beta+\left(a f_{x_{1}}+b f_{x_{2}}+c f_{x_{3}}\right) \sin \Omega= & \left(b f_{x_{3}} \cos \Omega-c f_{x_{2}} \cos \Omega\right) \frac{d x_{1}}{d s} \\
& +\left(c f_{x_{1}} \cos \Omega-a f_{x_{3}} \cos \Omega\right) \frac{d x_{2}}{d s} \\
& +\left(a f_{x_{2}} \cos \Omega-b f_{x_{1}} \cos \Omega\right) \frac{d x_{3}}{d s}
\end{aligned}
$$

can be written. By applying necessary operations to this last equation and (3.6) side by side, we obtain

$$
\frac{d x_{1}}{d s}=\frac{1}{\lambda}\left[\begin{array}{c}
f_{x_{2}}\left(\|\nabla f\| \cos \beta+\left(a f_{x_{1}}+b f_{x_{2}}+c f_{x_{3}}\right) \sin \Omega\right) \\
+\left(b \cos \Omega f_{x_{1}} f_{x_{2}}-a \cos \Omega f_{x_{2}}{ }^{2}+c \cos \Omega f_{x_{1}} f_{x_{3}}-a \cos \Omega f_{x_{3}}{ }^{2}\right) \frac{d x_{3}}{d s}
\end{array}\right]
$$

and

$$
\frac{d x_{2}}{d s}=\frac{1}{\lambda}\left[\begin{array}{l}
-f_{x_{1}}\left(\|\nabla f\| \cos \beta+\left(a f_{x_{1}}+b f_{x_{2}}+c f_{x_{3}}\right) \sin \Omega\right) \\
+\left(a \cos \Omega f_{x_{1}} f_{x_{2}}-b \cos \Omega f_{x_{1}}{ }^{2}+c \cos \Omega f_{x_{2}} f_{x_{3}}-b \cos \Omega f_{x_{3}}{ }^{2}\right) \frac{d x_{3}}{d s}
\end{array}\right]
$$

where $\lambda=f_{x_{2}}\left(b \cos \Omega f_{x_{3}}-c \cos \Omega f_{x_{2}}\right)+f_{x_{1}}\left(a \cos \Omega f_{x_{3}}-c \cos \Omega f_{x_{1}}\right) \neq 0$. Substituting the equations (3.8) and 3.9 into the equation (3.7) yields the quadratic 
equation with respect to $\frac{d x_{3}}{d s}$ as

$$
\left(B^{2}+D^{2}+1\right)\left(\frac{d x_{3}}{d s}\right)^{2}+(2 A B+2 C D) \frac{d x_{3}}{d s}+\left(A^{2}+C^{2}-1\right)=0
$$

where

$$
\begin{aligned}
A & =\frac{f_{x_{2}}\left(\|\nabla f\| \cos \beta+\left(a f_{x_{1}}+b f_{x_{2}}+c f_{x_{3}}\right) \sin \Omega\right)}{\lambda} \\
B & =\frac{b \cos \Omega f_{x_{1}} f_{x_{2}}-a \cos \Omega f_{x_{2}}{ }^{2}+c \cos \Omega f_{x_{1}} f_{x_{3}}-a \cos \Omega f_{x_{3}}{ }^{2}}{\lambda} \\
C & =\frac{-f_{x_{1}}\left(\|\nabla f\| \cos \beta+\left(a f_{x_{1}}+b f_{x_{2}}+c f_{x_{3}}\right) \sin \Omega\right)}{\lambda} \\
D & =\frac{a \cos \Omega f_{x_{1}} f_{x_{2}}-b \cos \Omega f_{x_{1}}{ }^{2}+c \cos \Omega f_{x_{2}} f_{x_{3}}-b \cos \Omega f_{x_{3}}{ }^{2}}{\lambda} .
\end{aligned}
$$

From the equation 3.10, we get

$$
\frac{d x_{3}}{d s}=\frac{(-2 A B-2 C D) \pm \sqrt{(2 A B+2 C D)^{2}-4\left(B^{2}+D^{2}+1\right)\left(A^{2}+C^{2}-1\right)}}{2\left(B^{2}+D^{2}+1\right)} .
$$

If the equation (3.11) is substituted into the equations 3.8 and 3.9 , an explicit 1st order ordinary differential equation system is found. Consequently, together with the initial point

$$
\left\{\begin{array}{l}
x_{1}(0)=x_{1}^{*} \\
x_{2}(0)=x_{2}^{*} \\
x_{3}(0)=x_{3}^{*}
\end{array}\right.
$$

we get an initial value problem. The solution of this problem yields the desired slant helical trajectory (according to PAFORS) on $M$.

Remark 3. Considering the obtained results, we can say followings:

(1) If $(2 A B+2 C D)^{2}-4\left(B^{2}+D^{2}+1\right)\left(A^{2}+C^{2}-1\right)<0$ at $\left(x_{1}^{*}, x_{2}^{*}, x_{3}^{*}\right)$, in that case any slant helical trajectory (according to PAFORS) on $M$ with the given fixed direction $\mathbf{g}$ and angle $\beta$ does not exist.

(2) If $(2 A B+2 C D)^{2}-4\left(B^{2}+D^{2}+1\right)\left(A^{2}+C^{2}-1\right)=0$ at $\left(x_{1}^{*}, x_{2}^{*}, x_{3}^{*}\right)$, in that case we have only one slant helical trajectory (according to PAFORS) on $M$ which passes through the initial point and accepts the given fixed unit direction $\mathbf{g}=(a, b, c)$ as an axis and the given angle $\beta$ as the constant angle.

(3) If $(2 A B+2 C D)^{2}-4\left(B^{2}+D^{2}+1\right)\left(A^{2}+C^{2}-1\right)>0$ at $\left(x_{1}^{*}, x_{2}^{*}, x_{3}^{*}\right)$, in that case we have two slant helical trajectories (according to PAFORS) on $M$ which pass through the initial point and accept the given fixed unit direction $\mathbf{g}=(a, b, c)$ as an axis and the given angle $\beta$ as the constant angle.

\section{Conclusion}

For a particle moving on a regular surface of $E^{3}$, there is a very close relationship between the kinematics of the particle, the differential geometry of the surface, and the differential geometry of the trajectory. As a result of this relationship, moving frames have been used as very useful tools to investigate the concepts of various studies in differential geometry and particle kinematics. PAFORS (Positional Adapted Frame on Regular Surface) has been recently developed for the trajectories having non-vanishing angular momentum by using the own position vector of 
the moving particle in 8 . It is expected that PAFORS will be widely preferred to discuss many special topics in particle kinematics and differential geometry. The present paper can be seen as the first step of these future studies.

In this paper, the geodesic, asymptotic, and slant helical trajectories are studied according to PAFORS in three-dimensional Euclidean space and some characterizations are given for them. Furthermore, we explain how we determine the helix axis for slant helical trajectories (according to PAFORS). Finally, we give a method to find the slant helical trajectory (if exists) lying on a given implicit surface which accepts a given fixed unit direction as an axis and a given angle as the constant angle.

In the future study, we plan to give MATLAB examples on the use of MATLAB command ODE45 to investigate the specific applications of the aforementioned method related to slant helical trajectories.

\section{REFERENCES}

[1] B. O'Neil, Elemantary differential geometry, Academic Press, Newyork, 1966.

[2] F. Dog̃an and Y. Yayl, Tubes with Darboux frame, Int. J. Contemp. Math. Sci., 7(16) (2012), 751-758.

[3] S. Kizlltuğ and Y. Yaylı, Timelike tubes with Darboux frame in Minkowski 3-space, International Journal of Physical Sciences, 8(1) (2013), 31-36.

[4] Ö. Bektaş and S. Yüce, Smarandache curves according to Darboux frame in $E^{3}$, Romanian Journal of Mathematics and Computer Science, 3(1) (2013), 48-59.

[5] B. Altunkaya and F. K. Aksoyak, Curves of constant breadth according to Darboux frame, Communications Faculty of Sciences University of Ankara Series A1 Mathematics and Statistics, 66(2) (2017), 44-52.

[6] G. Y. Şentürk and S. Yüce, Bertrand offsets of ruled surfaces with Darboux frame, Results in Mathematics, 72(3) (2017), 1151-1159.

[7] T. Körpınar and Y. Ünlütürk, An approach to energy and elastic for curves with extended Darboux frame in Minkowski space, AIMS Mathematics, 5(2) (2020), 1025-1034.

[8] K. E. Özen and M. Tosun, A new moving frame for trajectories on regular surfaces, Ikonion Journal of Mathematics, 3(1) (2021), 20-34.

[9] T. Shifrin, Differential geometry: A first course in curves and surfaces, University of Georgia, Preliminary Version, 2008.

[10] S. Izumiya and N. Takeuchi, New special curves and developable surfaces. Turk. J. Math., 28(2) (2004), 153-163.

[11] B. Bükcü and M. K. Karacan, The slant helices according to Bishop frame, Int. J. Comput. Math. Sci., 3(2) (2009), 67-70.

[12] A. T. Ali and M. Turgut, Some characterizations of slant helices in the Euclidean space $E^{n}$. Hacet. J. Math. Stat., 39(3) (2010), 327-336.

[13] O. Z. Okuyucu, İ. Gök, Y. Yayl and N. Ekmekci, Slant helices in three dimensional Lie groups, Appl. Math. Comput., 221 (2013), 672-683.

[14] P. Lucas and J. A. Ortega-Yagues, Helix surfaces and slant helices in the three-dimensional anti-De Sitter space. RACSAM, 111(4) (2017), 1201-1222.

[15] N. Macit and M. Düldül, Relatively normal-slant helices lying on a surface and their characterizations, Hacet. J. Math. Stat., 46(3) (2017), 397-408.

[16] K. E. Özen and M. Tosun, A new moving frame for trajectories with non-vanishing angular momentum, Journal of Mathematical Sciences and Modelling, 4(1) (2021), 7-18.

Kahraman EsEn ÖZEn,

Sakarya, Turkey, Orcid ID: 0000-0002-3299-6709

Email address: kahraman.ozen1@ogr.sakarya.edu.tr 
Murat Tosun,

Department of Mathematics, Faculty of Science and Arts, Sakarya University, Sakarya,

TURKEY, ORCID ID: 0000-0002-4888-1412

Email address: tosun@sakarya.edu.tr 